\title{
Diagnostic accuracy of abdominal compression for predicting fluid responsiveness in children.
}

Matthias Jacquet-Lagrèze, Nicolas Tiebergien, Najib Hanna, Sonia Courtil-Teyssedre, Jean-Noël Evain, Marc Lilot , Florent Baudin, Laurent Chardonnal , Dominique Bompard, Aurélie Portefaix , Etienne Javouhey, Jean-Luc Fellahi

Hôpital Cardiologique Louis Pradel - Hôpital femme mère enfant - Université de Claude Bernard Lyon

Backgroud and goal of Study:Administration of fluid to increase cardiac output is a cornerstone of the hemodynamic resuscitation. The purpose of this study was to evaluate the clinical usefulness of assessing variation of stroke volume during a calibrated abdominal compression for the prediction of fluid responsiveness in children.

Materials and methods: This study was approved by local institutional review boards (CPP Lyon Sud Est II). Patient, less than eight years with circulatory failure were selected. Stroke volume index was assessed with an echocardiography at baseline, after an abdominal compression (with a calibrated pressure of $25 \mathrm{~mm} \mathrm{Hg}$ ), at a return to baseline, and after a volume expansion $(10 \mathrm{ml} / \mathrm{kg}$ of fluid challenge over 10 minutes). Respiratory aortic blood flow velocity (Peak), and respiratory vena cava diameter variation (VClvar) were also recorded before volume expansion. Patients were classified as responders to fluid loading if their stroke volume index (SVI) increased by at least 15\%. R software with PROC package was used to performed descriptive and analytic statistic. $p<0.05$ was considered significant.
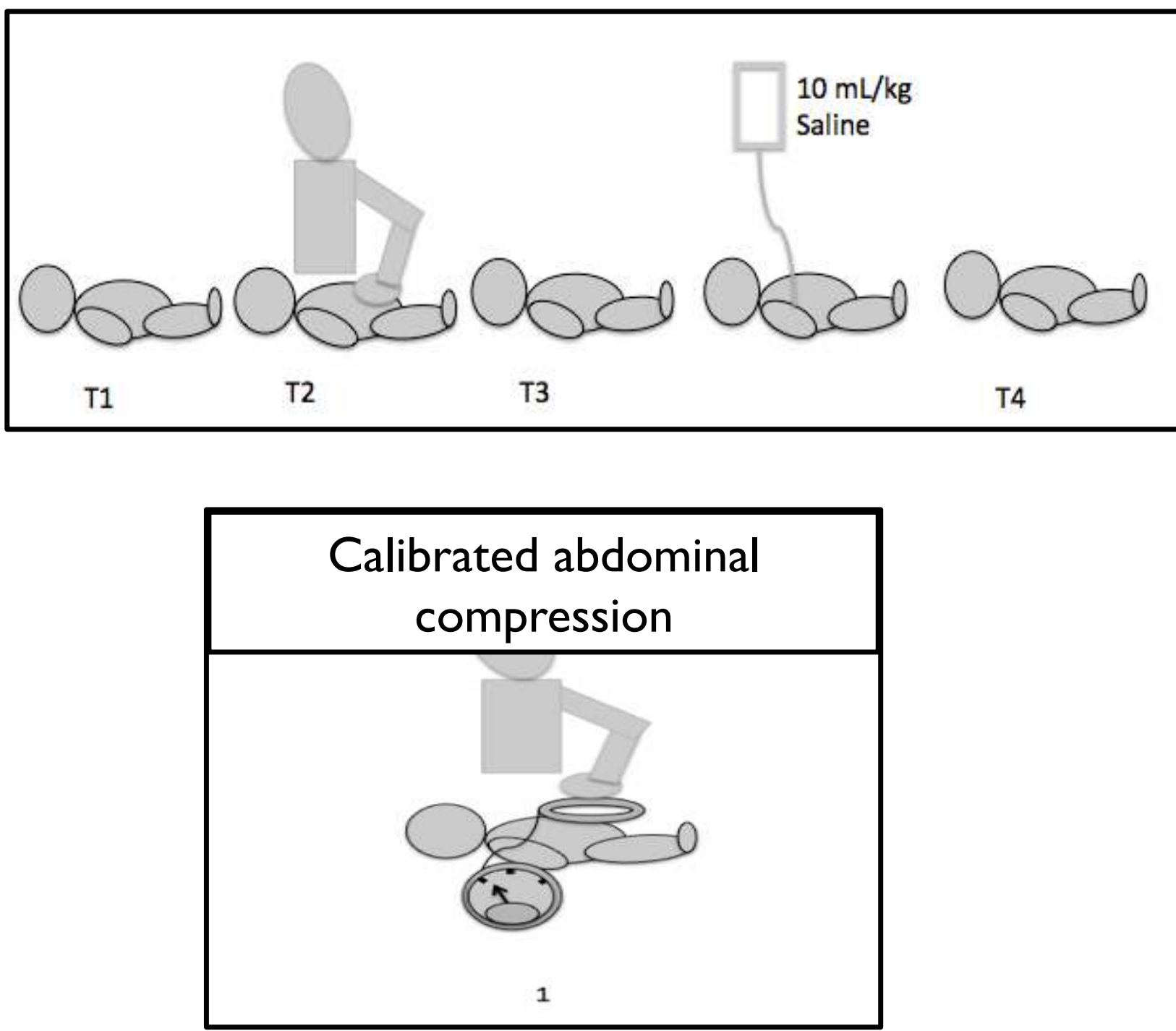

Results and Discussion: Thirty one children were included, 17( \pm $22)$ month old and weighing $8( \pm 5) \mathrm{kg}$. Seven were not on mechanical ventilation and 15 were on a mode allowing spontaneous breathing. 16 patients were fluid responders and 15 non-responders. Operators were blind from the value of the VTI. The least significant change of five averaged SVI was 10.6 (Cl: 6.8-I4.5) \% Change in SVI during abdominal compression and after a fluid challenge were correlated $\left(R^{2}=0.796\right.$ $\mathrm{P}<0.00 \mathrm{I})$. The ROC curve analysis showed that SVI change during abdominal compression predicts fluid responsiveness. (AUC ROC= $0.93 ; \mathrm{Cl}: 0.82-0.99)$. Best threshold was $9.28 \%$ with a sensitivity $75 \%(\mathrm{Cl}$ : $0.50-0.94)$ and a specificity of $93 \%(\mathrm{Cl}: 79-100)$. AUROC of VClvar was 0.70 (Cl: 0.48-0.9I). AUC ROC of delta Vpeak was 0.58 (Cl: 0.34-0.8I). Fluid responsiveness assessment, is challenging when spontaneous breathing is authorized [I]. It represents the vast majority of children hospitalized in PICU for acute circulatory failure. Our method seems quite reliable to predict fluid responsiveness, It was shown to be

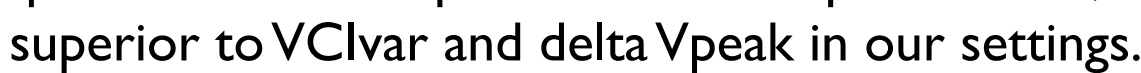

\section{Refernces:}

I. Gan H, Cannesson M, Chandler JR, Ansermino JM. Predicting Fluid Responsiveness in Children:A Systematic Review. Anesthesia \& Analgesia 2013 ;117:1380-92.
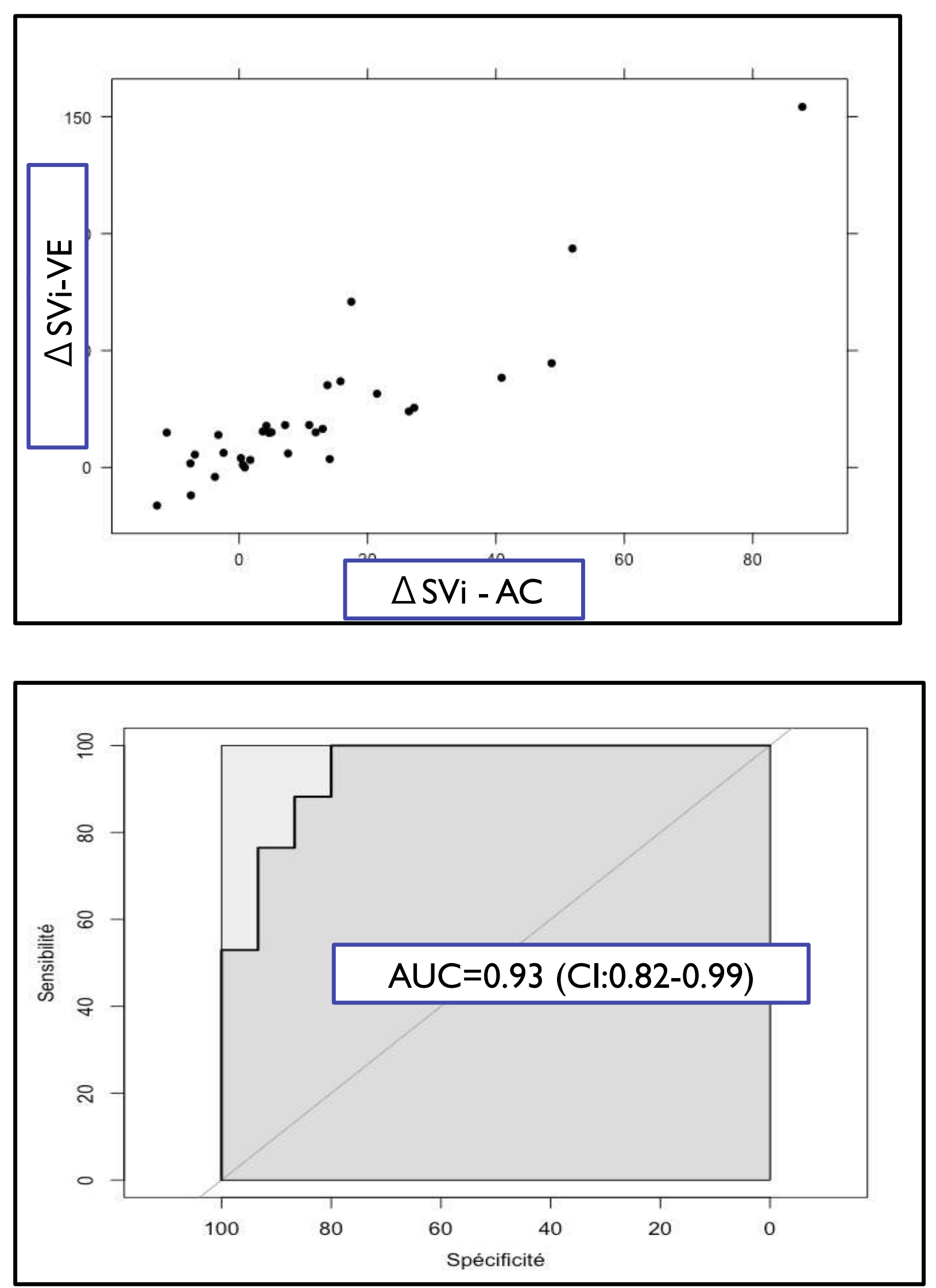

Conclusion: SVI variation during abdominal compression was the sole reliable method to predict fluid responsiveness in a mixed population of children, with and without spontaneous breathing, suffering from acute circulatory failure.
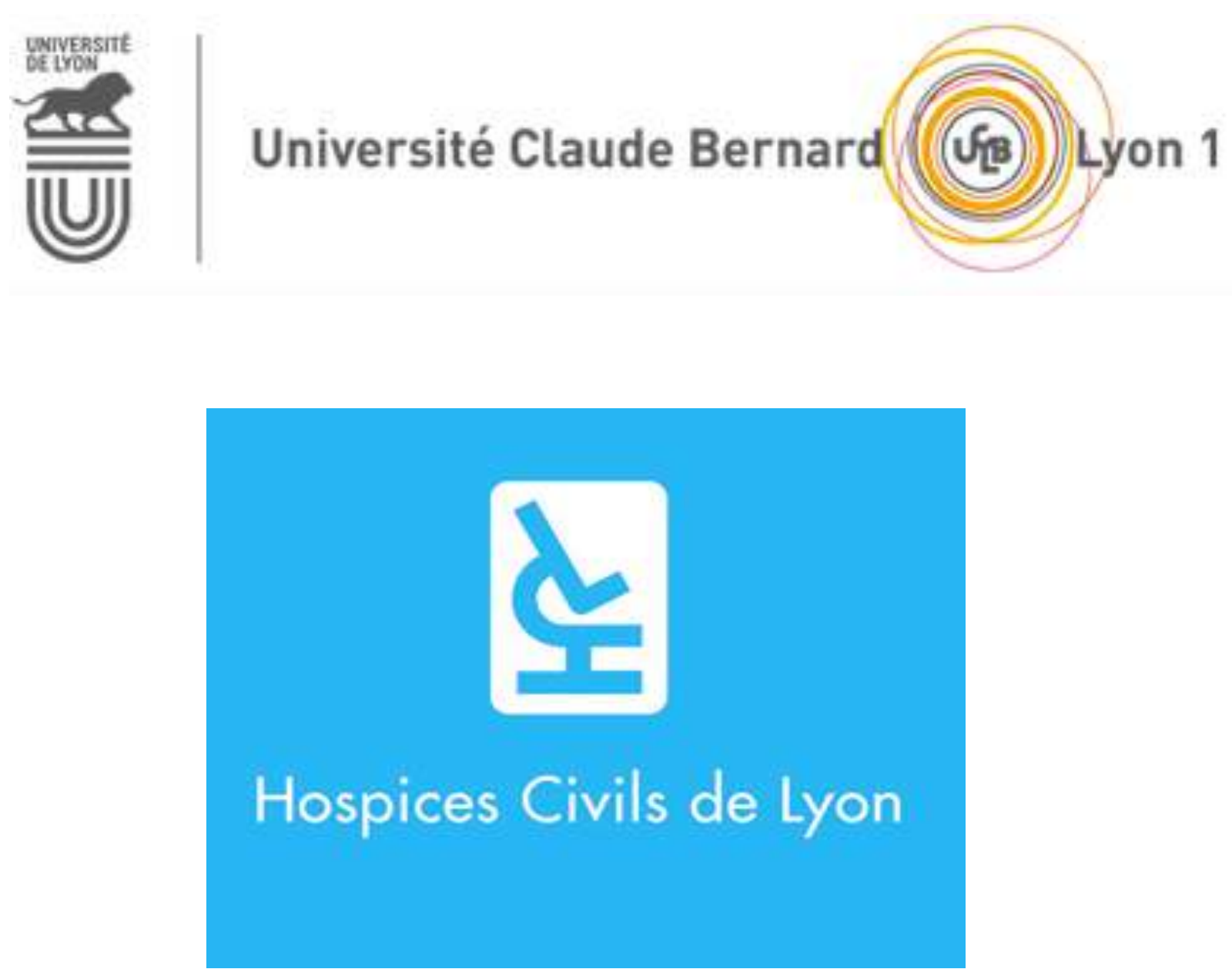